Rev. Biol. Trop., 47(3): 329-338, 1999

www.ucr.ac.cr www.ots.ac.cr www.ots.duke.edu

\title{
Weeds as a source for human consumption. A comparison between tropical and temperate Latin America
}

\author{
Martha Díaz-Betancourt ${ }^{1}$, Luciana Ghermandi ${ }^{2}$, Ana Ladio $^{2}$, Ismael R. López-Moreno ${ }^{1}$, Estela \\ Raffaele ${ }^{2}$ and Eduardo H. Rapoport ${ }^{2,3}$ \\ 1 Instituto de Ecología, Ap. Postal 63, Xalapa 91000, Veracruz, México. martha@ecologia.edu.mx \\ 2 Universidad Nacional del Comahue, Departamento de Ecología, 8400 Bariloche, Argentina and CONICET \\ rapoport@cab.cnea.edu.ar \\ 3 The sequence of authors was alphabetically ordered.
}

Received 16-IX-1998. Corrected 15-II-1999. Accepted 22-II-1999.

\begin{abstract}
Weeds abound in urban and agricultural environments. Depending on region and site, up to $66 \%$ of weed species are edible, and may constitute an additional food source for humans. Based on 400 samples, $1 / 4 \mathrm{~m}^{2}$ each, collected in tropical areas (e.g., roadsides, urban vacant lots, streets, sugar cane and coffee plantations in Coatepec, Mexico), average figures of edible fresh biomass vary between 1277 and $3582 \mathrm{~kg} / \mathrm{ha}$. A similar survey performed in a temperate area (739 samples in Bariloche, Argentina) showed mean values between 287 and $2939 \mathrm{~kg} / \mathrm{ha}$. A total of 43 species were sampled in Coatepec and 32 species in Bariloche. The general means were 2.1 and 1.3 tons/ha, respectively. At a greater geographic scale, a comparison between Mexican and Argentine weeds shows that, proportionately, the food parts vary a little between regions. In general, from higher to lower, the order of uses goes from leaves, seeds, roots, fruits, herbals, flowers and condiments. Edible roots (including bulbs and rhizomes) appear to be more common among perennials than among annuals.
\end{abstract}

Key words: Argentina, Bariloche, Coatepec, edible weeds, food plants, gathering, Mexico, Patagonia, urban flora.

Although plants have sustained the hunting and gathering peoples since the Paleolithic, the prevailing knowledge about edible species began to be lost since the invention of agriculture in the Neolithic. Two wellpreserved mummies found in Denmark, provided interesting information on food habits during the Iron Age. Their last meals contained 66 different plant taxa (Godwin 1960; King 1966), i.e., a diet much more diversified than that of modern man. According to FAO's Production Yearbooks, developed countries, and especially urban populations, began to depend almost entirely on the extensive and intensive agricultural production consisting in merely a little more than 100 food plants. Part of the old tradition is still maintained in some Latin American and eastern Asian countries. In Mexico, more than 20 "weeds" are also cultivated (Linares and Aguirre 1992). For instance, in Korean local markets 112 wild plants are sold at prices higher than those of cultivated species. Moreover, eleven species (some of them "weeds") are exported to the U.S.A. and used to prepare Korean and Chinese typical dishes (Pemberton and Lee 1996). Similarly, Moroccan weeds are exported with the same purpose to the U.S.A., Spain, Italy and Greece (Tanji and Nassif 1995).

The use of edible wild plants and weeds has been considered by several authors (Harris 1969, Kunkel 1984, Facciola 1990, Zurlo and 
Brandão 1990, Duke 1992, Linares and Aguirre, eds. 1992, and references there). Some of these books only provide extensive lists of species, with indications of their edible parts, while others add descriptions and illustrations of the species. Clarke (1977), Michael (1980), and Linares and Aguirre (1992) include numerous recipes. Edible weeds, however, are scarcely used in many countries, and weed gathering is more of a weekend hobby than a regular source of food supply.

To our knowledge, no attempts have been made to assess quantitatively the potential amount of food provided by common weeds and escapes.

Our interest was to evaluate the available biomass of human food provided by weeds in urban, periurban and field habitats in the northern and southern Neotropical region. We tried to compare two samples obtained in different climatic regions, i.e., tropical (Coatepec, Mexico) and temperate to cold-temperate (Bariloche, Argentina) areas.

\section{MATERIALS AND METHODS}

The environments selected were abandoned fields, main (paved) roads, secondary roads (suburban dirt roads), pathways, vacant urban and suburban lots. Sugar cane fields, milpas, coffee plantations (only Coatepec), and orchards (only Bariloche) were also considered. In these cases, we gathered and weighed all the edible parts of species appearing in 0.25 $\mathrm{m}^{2}$ quadrats (50 x 50 wooden frames). Along roadsides (haphazardly selected), we sampled at repeated $1 \mathrm{~km}$ distances, by laying ten quadrats regularly at $2 \mathrm{~m}$ distance each, parallel to the pavement. Urban vacant lots and fields were randomly sampled. The frames were laid along a random walk using a stopwatch to select compass direction and number of steps. The collected plants of each sample were kept in plastic bags and transported to the laboratory, separated by species and their edible portions were weighed with $0.01 \mathrm{~g}$ precision. Water content was calculated after $72 \mathrm{hr}$ dehydration at $60-80^{\circ} \mathrm{C}$ (Rapoport et al. 1995). Additionally, in some cases, the yields were assessed in terms of biomass collected per minute of harvesting. This survey was performed in order to evaluate whether there is any profit in gathering a given species in sites where it is abundant. In this way, we were in the position of a person searching for food. The analysis of the proportions of edible parts (roots, leaves, fruits, seeds) of all Mexican and Argentine weed species was obtained from Kunkel's (1984) list, and from our own records. Comparisons of fresh edible weights among habitats were made by means of Kruskal-Wallis tests. The comparison of total fresh weights between Mexico and Argentina was made by means of a Mann-Whitney test. In the case of significant differences, the Student-NewmanKeuls' test of multiple comparisons was applied. The detailed statistical analysis of data is reported elsewhere (Ladio et al. 1998).

The main roads sampled in Bariloche were roads No. 258 (Bariloche-El Bolsón), No. 237 (Bariloche-Alicura and Bariloche-Airport) and No. 237 (Bariloche-Llao Llao). In Coatepec, the road sampled was Carretera Briones.

\section{CLIMATE}

Comparative data on climates appear in Table 1.

\section{RESULTS}

The majority of the sampled species appear in Kunkel's (1984) list of food plants, to which we added Osmorhiza chilensis, from Argentina, and Drymaria gracilis, Galinsoga quadriradiata, Hydrocotyle bonariensis, Hydrocotyle mexicana, Margaranthus sulphureus, Oxalis latifolia, Sida glabra, S. spin$o s a$, and Tripogandra, serrulata from Mexico. These plants are commonly consumed by people and were repeatedly tasted by us.

In total, 43 species were recorded in Coatepec and 32 species in the Bariloche samples 
TABLE 1

Comparative characteristics of both areas

\begin{tabular}{|c|c|c|}
\hline & Coatepec & Bariloche \\
\hline Altitude & $1,252 \mathrm{~m}$ & $750 \mathrm{~m}$ \\
\hline Latitude & $19^{\circ} 27^{\circ} \mathrm{N}$ & $41^{\circ} 08^{\prime} \mathrm{S}$ \\
\hline Longitude & $96^{\circ} 57^{\circ} \mathrm{W}$ & $71^{\circ} 08-71^{\circ} 36^{\prime} \mathrm{W}$ \\
\hline Mean annual precipitation & $2250 \mathrm{~mm} *$ & $1000 \mathrm{~mm}^{* * *}$ \\
\hline Mean annual temperature & $18.8^{\circ} \mathrm{C}$ & $8.4^{\circ} \mathrm{C}$ \\
\hline Coldest month mean temp. & $15.2^{\circ}(\mathrm{Jan})$. & $2.3^{\circ}$ (July) \\
\hline Hottest month mean temp. & $21.4^{\circ} \mathrm{C}$ (May \& June) & $14.5^{\circ} \mathrm{C}$ (Feb.) \\
\hline Extreme temperature & 3.9 to $33.5^{\circ} \mathrm{C}$ & -18 to $35.5^{\circ} \mathrm{C}$ \\
\hline Prevailing winds & $\begin{array}{c}\text { N \& NW (winter), E, S \& SE } \\
\text { (spring, summer \& fall) }\end{array}$ & W \\
\hline Climate & A C (fm) w"a(i')g & $\begin{array}{c}\text { GCs lk (Mediterranean } \\
\text { regime) }\end{array}$ \\
\hline Population & 40,000 & 100,000 \\
\hline Source & Gómez and Soto 1990 & $\begin{array}{c}\text { Rudloff 1981; Grigera et al. } \\
\text { 1989; UNC } 1983\end{array}$ \\
\hline
\end{tabular}

(Tables 2 and 3). They represent, however, a small fraction of the real richness in both areas. More than $24 \%$ of the 700 weeds listed in Mexico by anonymous (1991) are edible. Of the 320 exotic weeds recorded in northwestern Patagonia (Rapoport and Brión 1991), 90 species $(28 \%)$ are edible.The Catalogue of Mexican Weeds (anonymous 1991) includes 168 edible species of which $36.3 \%$ are perennial and $63.7 \%$ annual and/or biennial. Similarly, in Argentina, the proportions are $35.0 \%$ and $65.0 \%$, respectively, from a total of 160 edible weeds that are mentioned in Marzocca et al. (1976). Perennials show a significantly higher proportion of edible roots (including tubers or rhizomes) and herbals than among the annualbiennia species $(P=0.027$ and 0.021 , respectively; Table 4). In contrast, annual-biennials show a greater proportion of edible leaves and seeds than perennials $(P=0.008$ and 0.012 , respectively) as showed by means of a $2 \times 2$ contingency table.

Yields per hectare: Because the number of sites surveyed and the number of samples per site were numerous, we made a tentative assessment of the amount of food ava lable per hectare (Table 5). In the case of plantations and orchards, cultivated plants were not included.
Coatepecan yields (2.1 tons, on average, per hectare) were apparently higher than the Barilochean yields (1. 3 tons per hectare). The extreme maximum yield was recorded on a main road between Coatepec and Xalapa $(10,158.0 \mathrm{~kg} / \mathrm{ha})$ based on 10 samples taken in January 1996, and on a vacant lot in Bariloche $(7,542.9 \mathrm{~kg} / \mathrm{ha})$ based on 7 samples taken in December 1996. When comparing equivalent Coatepecan and Barilochean environments by means of a Mann-Whitney test, the differences were not significant $(\mathrm{P}>0.05)$. However, the differences in the median values among total yields per hectare of Mexico and Argentina were significant $(\mathrm{U}=880 ; \mathrm{P}=0.03$ )

Gathering harvest. Species' yields: The following data correspond to fresh weights of the edible parts obtained per species in a given period of time. Sites were selected for their special abundances, from the point of view of a person searching for food. The process of gathering in Mexico took 15 minutes for each sample (five samples per species) and the mean values are presented per minute (Table 6).

In Bariloche, only three species were evaluated. (i) Taraxacum officinale (8 sampling sites, 21 minutes total gathering time). Depending on its abundances, yields varied 
TABLE 2

Coatepec, Mexico. Mean available food (fresh weight in grams) per $0.25 \mathrm{~m}^{2}$ sample and frequencies (number of times each species was found in 100 samples); $S D=$ standard deviation

\begin{tabular}{|c|c|c|c|c|c|c|c|c|c|c|c|c|}
\hline \multirow[t]{2}{*}{ Species } & \multicolumn{3}{|c|}{ Vacant lots } & \multicolumn{3}{|c|}{ Dirt roads \& pathways } & \multicolumn{3}{|c|}{ Plantations } & \multicolumn{3}{|c|}{ Highways } \\
\hline & $\begin{array}{c}\text { mean } \\
\text { weight }\end{array}$ & S.D. & freq. & $\begin{array}{c}\text { mean } \\
\text { weight }\end{array}$ & S.D. & freq. & $\begin{array}{l}\text { mean } \\
\text { weight }\end{array}$ & S.D. & Freq. & $\begin{array}{c}\text { mean } \\
\text { weight }\end{array}$ & S.D. & freq \\
\hline Acalypha wilkesiana & - & - & - & - & - & - & 6.68 & 6.17 & 7 & 1.57 & 1.01 & 5 \\
\hline Amaranthus dubius & 0.93 & 1.02 & 3 & 50.60 & 49.45 & 3 & 0.40 & 0.00 & 1 & - & - & \\
\hline Amaranthus hybridus & 30.71 & 34.22 & 6 & 6.53 & 5.29 & 3 & - & - & - & 17.63 & 27.05 & 14 \\
\hline Amaranthus spinosus & 6.10 & 5.32 & 8 & 47.68 & 0.00 & 1 & - & - & - & - & - & \\
\hline Anagallis arvensis & 1.23 & 0.90 & 2 & 0.84 & 0.46 & 4 & 0.27 & 0.00 & 1 & 2.35 & 1.62 & 8 \\
\hline Bidens odorata & 9.32 & 10.36 & 30 & 5.20 & 6.26 & 24 & 5.69 & 5.97 & 14 & 5.86 & 7.57 & 31 \\
\hline Brassica rapa & - & - & - & - & - & - & 3.07 & 2.30 & 4 & - & - & - \\
\hline Canna indica & - & - & - & 16.23 & 0.00 & 1 & - & - & - & - & - & \\
\hline Chenopodium ambrosioides & - & - & - & 59.32 & 0.00 & 1 & 4.03 & 0.00 & 1 & 13.29 & 0.00 & \\
\hline Commelina diffusa & 5.77 & 5.44 & 48 & 5.16 & 6.66 & 43 & 11.19 & 14.76 & 59 & 45.20 & 56.56 & 59 \\
\hline Commelina erecta & 20.90 & 14.25 & 3 & 12.21 & 6.82 & 5 & 34.67 & 46.29 & 40 & - & - & - \\
\hline Drymaria cordata & 5.32 & 6.65 & 27 & 1.31 & 1.46 & 15 & 5.92 & 7.73 & 24 & 0.92 & 0.45 & 2 \\
\hline Drymaria gracilis & 7.04 & 6.71 & 9 & 6.54 & 9.46 & 12 & 3.45 & 8.18 & 10 & 34.66 & 74.19 & 27 \\
\hline Galinsoga quadriradiata & 6.46 & 7.74 & 20 & 2.95 & 2.83 & 26 & 7.15 & 10.88 & 17 & 14.20 & 15.61 & 42 \\
\hline Heliconia caribaea & - & - & - & 342.81 & 204.63 & 3 & - & - & - & - & - & - \\
\hline Hydrocotyle bonariensis & 13.95 & 17.83 & 19 & 1.30 & 1.06 & 13 & 6.57 & 4.16 & 3 & - & - & - \\
\hline Hydrocotyle mexicana & 1.99 & 1.56 & 3 & 7.38 & 11.24 & 9 & - & - & - & - & - & - \\
\hline Ipomoea purpurea & - & - & - & - & - & - & - & - & - & 5.08 & 4.83 & 16 \\
\hline Ipomoea tilliacea & - & - & - & - & - & - & - & - & - & 5.09 & 4.38 & 7 \\
\hline Ipomoea triloba & 4.04 & 5.58 & 23 & 7.62 & 11.94 & 8 & 7.09 & 6.95 & 3 & 6.42 & 4.39 & 7 \\
\hline Margaranthus sulphureus & 6.72 & 7.87 & 6 & - & - & - & - & - & - & - & - & - \\
\hline Oxalis corniculata & 1.97 & 2.86 & 40 & 2.79 & 4.18 & 43 & 2.14 & 1.91 & 34 & 2.60 & 3.96 & 42 \\
\hline Oxalis latifolia & 1.70 & 1.61 & 14 & 4.92 & 5.75 & 17 & 9.28 & 16.28 & 45 & 3.43 & 4.87 & 20 \\
\hline Phaseolus vulgaris & - & - & - & - & - & + & - & - & - & 0.70 & 0.26 & 10 \\
\hline Piper auritum & - & - & - & 23.92 & 0.00 & 1 & - & - & - & 35.99 & 24.18 & 2 \\
\hline Plantago hirtella & - & - & - & 1.75 & 1.09 & 3 & - & - & - & 3.35 & 0.00 & 1 \\
\hline Plantago lanceolata & - & - & - & 158.62 & 0.00 & 1 & - & - & - & - & - & - \\
\hline Portulaca oleracea & + & - & - & - & - & - & 10.38 & 0.00 & 1 & 0.61 & 0.26 & 2 \\
\hline Rumex obtusifolius & 25.57 & 27.19 & 10 & 15.66 & 9.64 & 10 & 26.26 & 29.43 & 18 & 25.70 & 19.68 & 11 \\
\hline Sida acuta & - & - & - & - & - & - & 2.09 & 1.59 & 2 & - & - & \\
\hline Sida glabra & 1.33 & 0.00 & 1 & - & - & - & - & - & - & - & - & - \\
\hline Sida rhombifolia & 5.78 & 7.02 & 49 & 4.37 & 2.19 & 17 & - & - & - & 3.74 & 4.51 & 14 \\
\hline Sida spinosa & 2.87 & 2.26 & 10 & 14.05 & 12.59 & 8 & 5.41 & 6.87 & 4 & - & - & - \\
\hline Solanum nigrum & 3.75 & 3.62 & 3 & 9.70 & 0.00 & 1 & - & - & - & - & - & - \\
\hline Sonchus oleraceus & 8.99 & 2.05 & 2 & 22.13 & 14.96 & 3 & - & - & - & - & - & - \\
\hline Spilanthes americana & 10.24 & 10.75 & 34 & 13.52 & 23.17 & 20 & 15.65 & 14.97 & 30 & 12.68 & 17.63 & 32 \\
\hline Tagetes micrantha & - & - & - & - & - & - & + & - & - & 11.63 & 0.43 & 2 \\
\hline Taraxacum officinale & 0.39 & 0.00 & 1 & - & - & - & - & + & - & 6.41 & 5.61 & 4 \\
\hline Tradescantia fluminensis & 0.20 & 0.00 & 1 & 62.58 & 50.66 & 2 & 2.69 & 2.48 & 3 & 75.66 & 97.45 & 33 \\
\hline Trifolium repens & - & - & - & 2.51 & 3.29 & 14 & - & - & - & 8.39 & 7.19 & 5 \\
\hline Tripogandra serrulata & 13.27 & 28.39 & 22 & 10.71 & 13.54 & 11 & 20.88 & 20.08 & 55 & - & - & - \\
\hline Xanthosoma robustum & 16.53 & 15.85 & 10 & 14.48 & 16.79 & 10 & 18.68 & 13.15 & 7 & 10.08 & 8.30 & 7 \\
\hline Youngia japonica & 6.73 & 14.67 & 15 & 3.19 & 2.64 & 9 & 3.33 & 3.53 & 19 & 2.94 & 10.68 & 17 \\
\hline
\end{tabular}


TABLE 3

Bariloche, Argentina. Mean available food (fresh weight in grams) and frequencies (f) per $0.25 \mathrm{~m}^{2}$ sample. S.D.= standard desviation

Species

No. samples

Achillea millefolium

Alstroemeria aurea

Brassica rapa

Cichorius intybus

Cirsium vulgare

Claytonia perfoliata

Cytisus scoparius

Chenopodium album

Chrysanthemum

leucanthemum

Hypochoeris radicata

Lactuca serriola

Malus sylvestris

Malva sylvestris

Medicago lupulina

Melilotus albus

Mentha spp.

Oenothera odorata *

Osmorhiza chilensis *

Papaver rhoeas

Plantago lanceolata

Rumex acetosella

Rumex longifolius

Silybum marianum

Sonchus asper

Sonchus oleraceus

Stellaria media

Taraxacum officinale

Tragopogon dubius

Trifolium repens

* native species

\begin{tabular}{rrr}
\multicolumn{4}{c}{ Dirt roads \& } & Pathways \\
191 & & \\
mean & S.D. & f \\
weight & & \\
& & \\
0.04 & 0.13 & 1 \\
- & - & - \\
- & - & - \\
2.88 & 3.90 & 18 \\
- & - & - \\
- & - & - \\
- & - & - \\
- & - & - \\
3.16 & 6.36 & 5 \\
& & \\
0.80 & 0.30 & 3 \\
0.44 & 0.96 & 4 \\
0.21 & 0.65 & 1 \\
1.34 & 4.22 & 1 \\
0.22 & 0.71 & 1 \\
- & - & - \\
- & - & - \\
- & - & - \\
0.92 & 1.76 & 6 \\
0.17 & 0.54 & 1 \\
- & - & - \\
7.89 & 4.54 & 67 \\
1.60 & 1.24 & 25 \\
- & - & - \\
- & - & - \\
0.24 & 0.76 & 1 \\
- & - & - \\
- & - & - \\
- & - & - \\
3.25 & 3.89 & 27 \\
0.04 & 0.14 & 1 \\
0.05 & 0.16 & 1 \\
& &
\end{tabular}

Vacant lots

178

mean

mean S.D.

weight

$\begin{array}{rrr}- & - & \\ - & - & \\ - & - & \\ 2.83 & 4.73 & 26 \\ 1.39 & 3.10 & \\ 0.07 & 0.16 & \\ - & - & \end{array}$

$14.09 \quad 21.00^{-}$

$0.03 \quad 0.06$

$1.12 \quad 0.66$

$0.03 \quad 0.06$

$\begin{array}{lll}10.65 & 9.81 & 21\end{array}$

$\begin{array}{lll}2.65 & 2.89 & 28\end{array}$

$\begin{array}{rrr}- & - & - \\ - & - & - \\ 14.61 & 10.70 & 112\end{array}$

$\begin{array}{lll}3.08 & 2.46 & 34\end{array}$

$\begin{array}{lll}0.39 & 0.88 & 1\end{array}$

$0.55 \quad 1.24$

$\begin{array}{lll}0.12 & 0.27 & 1\end{array}$

$\begin{array}{rrr}2.21 & 4.72 & 7 \\ - & - & -\end{array}$

$\begin{array}{rrr}0.31 & 1.08 & - \\ 0.02 & 0.06 & 1 \\ 3.39 & 4.58 & 23 \\ - & - & - \\ - & - & - \\ 0.13 & 0.44 & 6 \\ 0.01 & 0.02 & 1 \\ 4.94 & 10.50 & 10 \\ & & \\ 2.65 & 4.99 & 24 \\ 0.10 & 0.29 & 4 \\ - & - & - \\ - & - & - \\ - & - & - \\ - & - & - \\ 0.29 & 0.71 & 2 \\ 10.14 & 34.48 & 2 \\ 0.04 & 0.14 & 2 \\ 0.72 & 1.54 & 6 \\ 0.01 & 0.02 & 1 \\ 2.28 & 1.54 & 28 \\ 3.38 & 4.83 & 22 \\ - & - & - \\ - & - & - \\ - & - & - \\ - & - & - \\ - & - & - \\ 4.40 & 15.19 & 1 \\ 4.14 & 4.54 & 30 \\ 0.03 & 0.10 & 1 \\ 0.01 & 0.04 & 1 \\ & & \end{array}$

Aband. fields

80

mean

weight

mean

S.D.

$\begin{array}{ll}0.39 & 1\end{array}$

$14.36^{-}$

10.99

0.
-

$0.35 \quad 0.77$

$21.71 \quad 14.87 \quad 36$

$\begin{array}{lll}1.49 & 3.34 & 2\end{array}$

$0.01 \quad 0.02$

$34.46 \quad 74.70$

$\begin{array}{lll}3.35 & 2.75 & 19\end{array}$

$1.02 \quad 1.21$ 


\section{TABLE 4}

A comparison of food parts in Mexican (168 species) and Argentine (160 species) weeds

\begin{tabular}{cccc}
\multicolumn{2}{c}{ México } & \multicolumn{2}{c}{ Argentina } \\
Perennials & Annuals/ & Perennials & Annuals/ \\
$(\%)$ & Biennials (\%) & $(\%)$ & Biennials (\%)
\end{tabular}

$\begin{array}{lrrrr}\text { Roots, rhizomes } & 15.4 & 7.7 & 19.1 & 8.0 \\ \begin{array}{l}\text { Leaves, stems, } \\ \text { buds }\end{array} & 30.7 & 44.0 & 41.2 & 54.0 \\ \text { Flowers } & 4.4 & 3.5 & 8.8 & 4.3 \\ \text { Fruits } & 13.2 & 10.5 & 8.8 & 2.2 \\ \text { Seeds } & 19.8 & 26.6 & 11.8 & 24.8 \\ \text { Condiments } & 4.4 & 2.8 & 4.4 & 5.1 \\ \text { Herbals } & 12.1 & 4.9 & 5.9 & 2.2\end{array}$

TABLE 5

Assessment of available food per hectare

\begin{tabular}{|c|c|c|c|}
\hline Coatepec & $\begin{array}{l}\text { No. samples } \\
\left(0.25 \mathrm{~m}^{2}\right)\end{array}$ & $\begin{array}{l}\text { Mean fresh } \\
\text { weight } \\
\text { yield (kg/ha) }\end{array}$ & sd \\
\hline \multicolumn{3}{|l|}{$\begin{array}{l}\text { Plantation (coffee, } \\
\text { sugar cane) }\end{array}$} & 1285.2 \\
\hline \multicolumn{4}{|l|}{ Urban \& suburban } \\
\hline vacant lots & 100 & 1277.1 & 663.5 \\
\hline Streets and pathways & 100 & 1453.4 & 1441.1 \\
\hline Main roads & 100 & 3582.4 & 3051.0 \\
\hline \multicolumn{4}{|l|}{ Bariloche } \\
\hline Abandoned fields & 80 & 287.2 & 219.2 \\
\hline \multicolumn{4}{|l|}{ Urban \& suburban } \\
\hline vacant lots & 193 & 1253.5 & 392.8 \\
\hline Orchards & 65 & 2938.8 & 3064.0 \\
\hline Streets and pathways & 191 & 1008.0 & 524.0 \\
\hline Main roads & 210 & 1326.6 & 460.0 \\
\hline
\end{tabular}

TABLE 6

Food yield (g) per minute in Coatepec, Mexico. Mean values based on 5 samples

$\begin{array}{lcccc}\text { Species } & \begin{array}{c}\text { Coffee } \\ \text { plantations }\end{array} & \begin{array}{c}\text { Sugar cane } \\ \text { plantations }\end{array} & \begin{array}{c}\text { Dirt } \\ \text { roads }\end{array} & \begin{array}{c}\text { Vacant } \\ \text { lots }\end{array} \\ \text { Bidens odorata } & - & 5.3 & - & - \\ \text { Commelina diffusa } & 11.8 & 12.8 & 13.8 & - \\ \text { Commelina erecta } & 18.2 & - & - & - \\ \text { Drymaria gracilis } & 14.6 & 10.8 & - & - \\ \text { Galinsoga quadriradiata } & 2.3 & - & - & - \\ \text { Heliconia caribaea } & - & - & 110.2 & - \\ \text { Hydrocotile bonariensis } & 4.8 & - & - & 1.7 \\ \text { Hydrocotyle mexicana } & - & - & 4.0 & - \\ \text { Oxalis latifolia } & - & - & 4.5 & - \\ \text { Rumex obtusifolius } & 23.8 & - & 37.8 & - \\ \text { Sida rhombifolia } & - & - & - & 5.0 \\ \text { Spilanthes americana } & 5.2 & 4.9 & - & 6.0 \\ \text { Xanthosoma robustum } & 33.2 & 29.4 & 15.0 & 21.6\end{array}$

between 9.1 and $36.4 \mathrm{~g} / \mathrm{min}$, with a mean of $17.0 \pm 8.8 \mathrm{~g} / \mathrm{min}$. (SD) of leaf blades without their central nerves. (ii) Chenopodium album (3 samples, 9 minutes gathering time). Yields were from 38.3 to $56.3 \mathrm{~g} / \mathrm{min}$, with a mean of $48.0 \pm 9.1 \mathrm{~g} / \mathrm{min}$. Only leaves and tender apical stems were considered. (iii) Montia perfoliata (11 sampling sites, 40 minutes total gathering time). Yields from 31.0 to $239.6 \mathrm{~g} / \mathrm{min}$, with a mean of $80.4 \pm 65.0 \mathrm{~g} / \mathrm{min}$. Leaving aside the figures obtained in Heliconia carib$a e a$, which represents a special case for its edible roots, yields seem more productive in Bariloche than in Coatepec. Outstanding biomasses are produced by Montia perfoliata, a North American invader of Patagonian urban forests.

\section{The most profitable plants in Bariloche: Montia perfoliata $(=$ Claytonia perfoliata $)$}

This species shows clear capabilities to recover after harvesting. During the 1995 growth season, in Bariloche we measured the fresh biomasses of three $0.25 \mathrm{~m}^{2}$ plots completely dominated by $M$. perfoliata. The procedure was repeated on the same plot ca. 30 and 60 days later, with the following results (in grams).

$\begin{array}{lcccc} & \text { October } & \text { November } & \text { December } & \text { Totals } \\ \text { Plot A } & 138.6 & 115.1 & 22.3 & 276.0 \mathrm{~g} \text {. } \\ \text { Plot B } & 487.9 & 141.9 & 96.7 & 726.5 \mathrm{~g} . \\ \text { Plot C } & 381.0 & 398.6 & 48.5 & 828.1 \mathrm{~g} \text {. }\end{array}$

On the basis of 34 samples where $M$. perfoliata was abundant, food biomasses varied from 108.5 to $973.6 \mathrm{~g}$ per $0.25 \mathrm{~m}^{2}$ plot. Mean value $275.9 \pm 210.6 \mathrm{~g}(\mathrm{SD})$. The aerial parts of this plant are $100 \%$ edible. Average water content varied around $78.5 \pm 7.3 \%$.

\section{Plantago lanceolata}

Leaf fresh weight per $0.25 \mathrm{~m}^{2}$ plots $(\mathrm{n}=$ 25 plots) showed figures ranging from 39.2 to 309.2 g. Mean value $124.1 \pm 60.2$ g. Seeds should be added to this food source. At the end of the summer, we selected three $1 \mathrm{~m}^{2}$ plots showing ripe fruits. On average, there were 
TABLE 7

Proportions of food plants in different biomes and communities

Natural Ecosystems

$\begin{array}{lrr}\text { Total } & \text { No. edible } \\ \text { No. spp. } & \text { spp. } & \% \\ 18,956 & 1,112 & \\ & & \\ 2,500 & 375 & 15 \\ 430 & 26 & \\ 360 & 75 & 2 \\ 275 & 11 & \end{array}$

Anthropic Ecosystems

\begin{tabular}{|c|c|c|c|}
\hline 653 & 124 & 19 & SW Córdoba Province, Argentina \\
\hline 446 & 77 & 17 & $\begin{array}{l}\text { W Uruguay, E Entre Ríos Prov., } \\
\text { Argentina, S Brazil (Salto Grande } \\
\text { Dam) }\end{array}$ \\
\hline 165 & 55 & 33 & Parque La Chata, La Habana, Cuba \\
\hline
\end{tabular}

North America. Food plants used by the aboriginal populations Sonoran Desert

Tierra del Fuego, Ona Indians (1) Bolivian Amazon

Peruvian Amazon (only fruit trees considered)

Source

Duke 1992*

Felger \& Nabhan 1978*

Martínez-Crovetto 1968

Boom 1987

Peters et al. 1989

Bianco \& Cantero 1992

Lema 1988

E.H. Rapoport, C.R. Martínez, P. Herrera (unpublished)

Strictly Weeds

$\begin{array}{rrrll}51 & 31 & 61 & \text { Experimental Field, Saskatchewan } & \text { Derksen } \text { et al. } 1993 \\ 14 & 6 & 43 & \text { Slash \& burn, NE India } & \text { Misra } \text { et al. } 1992 \\ 761 & 177 & 23 & \text { Weed Catalog, Argentina } & \text { Marzocca } \text { et al. } 1976 \\ 300 & 90 & 30 & \text { Weeds and escapes, NW Patagonia } & \text { Rapoport \& Brión 1991 } \\ 200 & 59 & 30 & \text { Random sample, World Weeds } & \text { Rapoport unpublished } \\ 40 & 20 & 50 & \text { Most common weeds of Cuba } & \text { Sánchez \& Huranga 1970 } \\ 176 & 51 & 31 & \text { Urban weeds, Rosario City, Argent. } & \text { Franceschi 1996 } \\ 168 & 11 & 66 & \text { Canadian weeds } & \text { Frankton \& Mulligan } 1977 \\ 422 & 177 & 42 & \text { U.S.A. weeds } & \text { Wilkinson \& Jaques } 1979 \\ 305 & 137 & 45 & \text { Xalapa City, Mexico } & \text { Domínguez-Barradas } 1995 \\ 18 & 16 & 89 & \text { World's most aggressive weeds } & \text { Holm } \text { et al. } 1977\end{array}$

* Data provided by the authors. The rest was assessed by us in Kunkel's (1984) list and our own records.

(1) Of the 9 Ona Indians extant only 6 were interviewed. They were very old people and only one woman used Shelknam language exclusively. Of the 430 native vascular species of the island 24 were used as food (5.6\%). Two additional native edible plants were ignored by the reporters. From 128 exotic species only Hypochaeris radicata and Taraxacum officinale were included in their diets, although there are 7 more species which appear as edible in Kunkel's list.

$356.0 \pm 38.4$ spikes $/ \mathrm{m}^{2}$ in high density patches. Each spike may contain about 500 seeds measuring $2 \mathrm{~mm}$ each, showing about $36 \%$ abortions. Viable seeds were assessed in $162.1 \mathrm{~g} / \mathrm{m}^{2}$. Although the process is laborious (about one hour-person to screen the seeds of one square meter), it is useful to know that a one hectare monospecific plot may yield more than 1.5 tons of seeds, in addition to almost 5 tons of leaves.
Of course, it would imply the design of adequate mechanical technology to accelerate the process of screening seeds. Average water content of leaves varied around $78.6 \pm 4.9 \%$.

\section{Rumex acetosella}

Yields vary between 8.9 and $186.7 \mathrm{~g} / 0.25$ $\mathrm{m}^{2}$. Mean $53.3 \pm 47.9 \mathrm{~g}(\mathrm{n}=15$ samples). The species is widely distributed in disturbed and 
undisturbed habitats, especially in surrounding grasslands and pasturelands. Average water content varied around $77.1 \pm 4.6 \%$.

\section{Taraxacum officinale}

Yields vary between 11.2 and $107.4 \mathrm{~g} / 0.25$ $\mathrm{m}^{2}$ (central ribs excluded). Mean 47.2 $\pm 27.6 \mathrm{~g}$ ( $\mathrm{n}=17$ samples). Average water content varied around $82.2 \pm 4.7 \%$.

\section{DISCUSSION}

Common weeds prove to be an interesting resource in small to medium-sized human settlements where they may provide supplementary food. In large cities, suburban populations may also profit from edible weeds. The data from Table 7 show that edible, non-weedy plants comprise between 6 and 21 percent of the biomass of the natural communities surveyed. The proportion of edible plants increases considerably in anthropic environments, especially in weed communities. Roughly, ten percent of the 260,000 known vascular species of the world should be considered as a potential source for human consumption. It is probably not by mere chance that the majority of the centers of origin or domestication of cultivated plants proposed by Vavilov (1938) corresponded to ancient, sedentary cultures. The idea that civilizations arose in areas with abundant edible plants adequate for culturing is probably incorrect. Since edible plants abound everywhere, it seems that civilizations developed in any environment where for historical reasons, people had time enough to exploit their natural resources in a more permanent and intensive way. By selection, plants originally wild, began to be more productive and adequate for human consumption. Rye, oats, carrots and several other cultivated plants originated as weeds, a fact that gives a clear idea of the enormous potential of weeds and other wild non-weedy plants as a source for new cultures. The economic incentive provided by the revival of ancient gastronomic traditions persuaded some private entrepreneurs to change from the occasional gathering to a more permanent cultivation of "weeds". Popular markets in Mexico (Linares and Aguirre 1992) and Korea (Pemberton and Lee 1990) offer a variety of gourmet "weeds" at higher prices than the common vegetables.

A case of human-livestock-plant mutualism is mentioned by Kuznar (1993), especially referred to species of Chenopodium proliferating in unusual concentrations in corrals. Herd animals transport these forage species to pastoral campsites where the plants thrive in the organic corral soils. This creates a mutually beneficial relationship where certain plant species become camp followers of pastoral campsites. This is the process by which plant invaders reach the status of weeds first and, later on, the status of cultivars, according to Vavilov (1938). And this process may explain the fact that the majority of the most aggressive and cosmopolitan weeds are edible. Initially, plant domestication began early, and in an unconscious way, probably in the Paleolithic Age, in primitive hunter-gatherer cultures (Rapoport et al. 1995). It is interesting to note that leaf-cutting ants (Acromyrmex), as determined by Farji-Brener (1996), show clear foraging preferences for exotic ruderal weeds which, according to Coley et al. (1985), are plants that invest more energy in reproduction, growth and dispersal than in anti-herbivore chemical defenses. It is suggestive that $52 \%$ of the early introductions (archaeophytes) in Poland listed by Trzcinska (1982) are edible.

Our results show that in anthropic habitats there are immense amounts of edible plants which are not always totally profited from. This is clearly evident in Argentina where people have almost lost the ancient practice of gathering wild food plants. In a tropical area such as Coatepec, the overall 'standing crop' averages 2.1 tons/ha whilst in temperate Bariloche it reaches 1.3 tons/ha. Tropical weeds are richer in species number and more productive than temperate weeds. The latter are almost absent during the long winter season, although they can be dehydrated and cooked during the cold season. 
Because a significant sector of the Argentine population suffers from serious problems of malnutrition, the Universidad del Comahue and Municipalidad de Bariloche published a booklet (Rapoport et al. 1997) illustrating the 20 most common edible weeds. Free copies were distributed in provincial public schools. This instruction manual represents the beginning of a program which hopes to restøre, at least partially, our ancestors' knowledge. As a result of this printed information and a television program, a substantial increment of popular awareness and utilization of this resource, was registered.

\section{ACKNOWLEDGEMENTS}

Our thanks to Andrea Premoli, Tom Witham and Barbara Drausal for their useful comments on the article.

\section{RESUMEN}

Las malezas abundan en ambientes urbanos y rurales. Según la región y lugar, hasta el $66 \%$ de las especies de malezas pueden ser comestibles y constituir un recurso alimentario adicional para el ser humano. Sobre la base de 400 muestras de $1 / 4 \mathrm{~m}^{2}$ cada una, recolectadas en áreas tropicales (rutas, terrenos balcríos, calles y plantaciones en Coatepec, Méxicol el promedio de la biomasa en peso fresco varió entre 1277 y $3582 \mathrm{~kg} / \mathrm{ha}$. Un muestreo similar en un área templada (739 muestras en Bariloche, Argentina) arrojó valores medios entre 287 y $2939 \mathrm{~kg} / \mathrm{ha}$. En total se registraron 43 especies en Codtepe y 32 especies en Bariloche. La media general (iotai) fue de 2.1 y 1.3 ton/ha, respectivamente. A una escala geogréfica mayor, una compuaración entre las malezas mexiçnas $\mathrm{y}$ argentinas no mostró mayores variaciones regionales en cuanto a qué partes u órganıs son los comestibles. En ambos lugeres, el orden de aprovechamiento, de mayor a menor, fue: hojas, semillas, raices, frutos, inítusiones, fores y condimentos. Las roices comestibles (ncluyendo balcos y rizomas) parecen ser más comunes entre las especies perennes que entre las norales.

\section{REFERENCES}

Anonymous. 199. Catálogo de especies vegetales consideradas malezas. Asoc. Mex. Ciencia de la Maleza, AMCMí Méxice, D.F. p. 92.
Bianco, C.A. \& J.J. Cantero. 1992. Las plantas vasculares del suroeste de la Provincia de Córdoba. Iconografía. Facultad Agronomía y Veterinaria. Universidad Nacional Río Cuarto, Rí Cuarto, Argentina. p. 229

Boom, B. M. 1987. Ethnobotany of the Chácobo Indians, Beni, Bolivia. Adv. Econ. Bot. 4: 1-68.

Clarke, C.B. 1977. Edible and useful plants of California. University of California, Berkeley, California. p. 280.

Coley, P.D., J.P. Briant \& F.S. Chapin. 1985. Resource availability and plant anti-herbivore defense. Science 230: $895-899$

Derksen, D. A., A. G. Thomas, G. P Lafond, H. A Loeppky \& C. J. Swanton. 1993. Impact of agronomic practices on weed communities: Fallow within tillage systems. Weed Sci. 42: 184-194.

Domínguez Barradas, B. A. 1995. La vegetación vascular espontánea en calles y banquetas de la Ciudad de Xalapa, Veracruz. Tesis Licenciatura, Universidad Veracruzana, Facultad de Biología, Veracruz, México. p. 93.

Duke, J. A. 1992. Handbook of edible weeds. CRC, Boca Raton, Florida. p. 246.

Facciola, S. 1990. Cornucopiá. A source book of edible plants. Kampong, Vista, California. p. 678.

FAO. 1987. Production yearbook. Food and Agriculture Organization, United Nations, Rome: 1-306.

Farji-Brener, A.G. 1996. Posibies vías de expansión de la hormiga cortadora de hojas Acromymex lobicornis haciz la P'atagonia. Ecologia Austral 6: 144-150.

Fe'gor, R.S., and G.P. Nabhan. 1978. Agroecosystem diversity: $A$ model from the Sonoran desert. AAAS (Am. Assoc. Adv. Sci.) Selected Symposia 10.

Franceschi, E. A. 1996. The ruderal vegetation of Rosario City, Argentina. Landscape Urb. Plann. 34: 11-18.

Frankton, C. \& G.A. Muiligen. 1977. Weeds of Canada. Canada Deptartment of . Agriculture, Publication 948. p. 217.

Godwin, H. 1969. The hiswry of weers in Britain. p. 1-10. In: The biology of weeds. J.L. Harper (ed.). Blackwell Oxford, U.K.

Gónez, C.M. \& M. Soto, 1990. Climático del Municipio de Coatepec. Instituto de Ecología, Publ. No. 27, Xalapa, Veracruz, México. 
Grigera, D., E. Bianchi, C. Brión, J. Puntieri \& N. Rodríguez. 1989. Carta del medio ambiente y su dinámica de San Carlos de Bariloche, 1987. Universidad Nacional del Comahue, Bariloche, Argentina. p. 40.

Harris, B.C. 1969. Eat the weeds. Keats Publishing Inc., New Canaan, Connecticut, p. 254.

Holm, L.G., D.L. Plucknett, J.V. Pancho \& J.P. Herberger. 1977. The world's worst weeds. University of Hawaii, Honolulu, Hawaii, p. 609.

King, L. W. 1966. Weeds of the world. Biology and Control. Leonard Hill, London. p. 526.

Kunkel, G. 1984. Plants for human consumption. Koeltz Scientific Books, Koenigsten, Germany. p. 393.

Kuznar, L.A. 1993. Mutualism between Chenopodium, herd animals, and herders in the south central Andes. Moun. Res. Dev. 13: 257-265.

Ladio, A.H., E. Raffaele, L. Ghermandi, M.E. DíazBetancourt, I. López-Moreno \& E.H. Rapoport. 1998. Las malezas en las ciudades de S.C. de Bariloche y Coatepec y su potencial oferta alimentaria. Actas XIII Congr. Latinoam. Malezas, Buenos Aires, Argentina.

Lema, O. W. 1988. Catálogo ilustrado de la flora de Salto Grande. Introducción a su estudio. Tomo I. Comisión Técnica Mixta de Salto Grande, Argentina-Uruguay. p. 312 .

Linares, E. \& J. Aguirre (eds.) 1992. Los quelites, un tesoro culinario. Universidad Autónoma de México \& Instituto Nacional de la Nutrición "Salvador Zubirán", México. p. 141.

Michael, P. 1980. All good things around us. A cookbook and guide to wild plants and herbs. Holt, Rinehart \& Winston, New York, p. 240.

Martínez-Crovetto, R. 1968. Estudios̀ etnobotánicos. IV. Nombres de plantas y su utilidad, según los indios onas de Tierra del. Fuego. Etnobiologica 3: 12-19.

Marzocca, A., O.J.V. Mársico \& O. Del Puerto. 1976. Manual de malezas. Hemisferio Sur, Buenos Aires, Argentina. p. 564.

Misra, J., H. N. Pandey, R. S. Tripathi \& U. K. Sahoo. 1992. Weed population dynamics under 'jhum' (slash and burn agriculture) and terrace cultivation in northeast India. Agric., Ecosyst. Env. 41: 285-295.

Pemberton, R. W. \& N. S. Lee. 1996. Wild food plants in South Korea: market presence, new crops, and exports to the United States. Econ. Bot. 50: 57-70.

Peters, C. M., A. H. Gentry \& R. O. Mendelsohn. 1989. Valuation of an Amazonian rainforest. Nature 339: 655-656.

Rapoport, E.H., \& C. Brión. 1991. Malezas exóticas y plantas escapadas de cultivo en el noroeste patagónico. Segunda Aproximación. Cuadernos de Alter Natura No. 1, Bariloche, Argentina. p. 19.

Rapoport, E.H., L.S. Margutti \& E.H. Sanz. 1997. Plantas silvestres comestibles de la Patagonia Andina. Parte I. Exóticas. Programa Extensión Universitaria, Univ. Nac. del Comahue, Depto. Ecología, CRUB, Bariloche, Argentina. p. 51.

Rapoport, E.H., E. Raffaele, L. Ghermandi \& L. Margutti. 1995. Edible weeds: A scarcely used resource. Bull. Ecol. Soc. Amer. 76: 163-166.

Rudloff, W. 1981. World-Climates. Wissenschaftliche Verlagsgesellschaft, Stuttgart, Germany. p. 632.

Sánchez, P. \& H. Huranga. 1990. Malezas importantes de Cuba. Instituto de Investigación Fundamental de Agricultura Tropical A. de Humboldt, Ministerio de Agricultura, Cuba. p. 63.

Tanji, A. \& Nassif, F. 1995. Edible weeds in Morocco. Weed Technol. 9: 617-620.

Trzcinska-Tacik, H. \& K. Wasylikowa. 1982. History of the synanthropic changes of flora and vegetation of Poland. Mem. Zool. 37: 47-69.

UNC. 1983. Cartas de precipitación de las provincias de Río Negro y Neuquén. Facultad Ciencias Agrarias, Universidad Nacional del Comahue, Cinco Saltos, Argentina.

Vavilov, N.I. 1938. Los centros genético-geográficos de las plantas cultivadas. Rev. Fac. Agron. Univ. Nac. La Plata 22: 65-94.

Wilkinson, R.E. \& H.E Jaques. 1979. How to know the weeds. W.C. Brown, Dubuque, Iowa p. 230.

Zurlo, C. \& M. Brandão. 1990. As ervas comestíveis. Globo, São Paulo, Brazil. p. 167. 